\title{
Perfil clínico de um paciente amputado transfemoral: relato de caso
}

\section{Clinical profile of a transfemural amputee patient: a case report}

\author{
Jéssica Franco Dalenogare ${ }^{1}$, Ana Paula Ziegler Vey¹, Jéssica Michelon Bellé ${ }^{1}$, Samantha Luise \\ Adami $^{1}$, Melissa Medeiros Braz ${ }^{1}$
}

1 - Universidade de Santa Cruz do Sul - UNISC, Santa Cruz do Sul, RS, Brasil.

2 - Universidade Federal de Santa Maria - UFSM, Santa Maria, RS, Brasil.

\begin{abstract}
RESUMO
Introdução: a amputação é a retirada total ou parcial de um membro. Em decorrência disso, algumas complicações podem influenciar negativamente a vida do indivíduo, como alteração da imagem corporal e redução da qualidade de vida. Desta forma, o conhecimento do perfil clínico destes pacientes é de fundamental importância para melhor compreensão e abordagem destes pacientes amputados. Objetivo: relatar o perfil clínico de um paciente amputado transfemoral unilateral. Método: relato de caso descritivo, realizado com um paciente no ambulatório de fisioterapia do Hospital Universitário de Santa Maria (HUSM). Para avaliação foram aplicados os testes Short Form-36 (SF-36), Timed Up and Go (TUG), Questionário Nórdico de Sintomas Osteomusculares e Body Image Scale (BIS). Resultados e Conclusão: por meio dos testes, observa-se que a amputação pode interferir de forma negativa na percepção e satisfação com a imagem corporal do sujeito, deixá-lo mais suscetível

Palavras-chave: a quedas além de queixas de dor e desconforto. Dessa forma, percebe-se que há a necessidaAmputação; Qualidade de de de uma avaliação fisioterapêutica mais abrangente dos pacientes amputados, que englobe Vida; Imagem Corporal; não somente aspectos físicos, mas também psicoemocionais, para desenvolver um olhar Dor Musculoesquelética. ampliado do indivíduo e consequentemente uma terapêutica efetiva e mais individualizada.
\end{abstract}

\begin{abstract}
Introduction: amputation is the total or partial removal of a limb. As a result, some complications can negatively influence the individual's life, such as altered body image and reduced quality of life. Thus, knowledge of the clinical profile of these patients is of fundamental importance for better understanding and approach of amputee patients. Objective: to report the clinical profile of a unilateral transfemoral amputee patient. Method: descriptive case report, performed with a patient at the physiotherapy outpatient clinic of the University Hospital of Santa Maria (HUSM). For evaluation, the Short Form-36 (SF-36), Timed Up and Go (TUG), and Nordic Musculoskeletal Symptoms Questionnaire and Body Image Scale (BIS) were applied. Results and Conclusion: through the tests, it is observed that amputation can negatively interfere in the individual's perception and satisfaction with their body image, make them more susceptible to falls in addition to complaints of pain and Keywords: discomfort. Thus, there is a need for a more comprehensive physical therapy evaluation of
\end{abstract} Amputation; Quality of amputee patients, which encompasses not only physical, but also psychoemotional aspects, Life; Body Image; Muscu- to develop an expanded view of the individual and, consequently, an effective and more loskeletal Pain. individualized therapy. 


\section{INTRODUÇÃO}

Amputação é a retirada total ou parcial de um membro. ${ }^{1}$ No Brasil, as amputações de membros inferiores correspondem a $85 \%$ de todas as amputações de membros, segundo informações do Ministério da Saúde. ${ }^{2}$ Este procedimento pode ocorrer como consequência de complicações de doenças crônico-degenerativas, como a diabetes, ou por causas traumáticas, dentre outros motivos. As amputações decorrentes de traumas são majoritariamente causadas por acidentes de trânsito e ferimentos por arma de fogo, sendo responsáveis por $20 \%$ das amputações de membros inferiores. ${ }^{3}$

Como consequência da amputação, algumas complicações podem influenciar a vida do indivíduo amputado. Estas condições vão além de questões puramente físicas, como redução da mobilidade, dor fantasma, dor musculoesquelética e problemas no equilíbrio. Nesse sentido, destacase o comprometimento da imagem corporal e da qualidade de vida, alterações que implicam em uma série de restrições e adaptações tanto para o sujeito quanto para sua família. Assim, a qualidade de vida é compreendida como a sensação de bem-estar com as esferas social, espiritual, física, mental, psicológica e emocional além de sensação de sucesso nos relacionamentos sociais do indivíduo, sendo percebida de forma individual para cada pessoa, de acordo com sua experiência de vida, valores e perspectivas. ${ }^{4}$

Neste contexto, a fisioterapia faz-se necessária no tratamento e acompanhamento destes pacientes, podendo contribuir não só para as melhoras na reabilitação física, mas também nos aspectos da qualidade de vida desses pacientes. ${ }^{3}$ Assim, para uma reabilitação eficiente é essencial que a avaliação fisioterapêutica do amputado seja feita de forma integral, abrangendo além das questões físicas também os aspectos psicológicos, emocionais e sociais. Dessa forma, o presente estudo objetiva traçar o perfil clínico de um paciente amputado transfemural, correlacionando as alterações na qualidade de vida e imagem corporal após a amputação, a fim de demonstrar a importância de uma avaliação amplas destes pacientes, associando seus aspectos físicos e psicoemocionais.

\section{MÉTODO}

Trata-se de um relato de caso descritivo com o objetivo de traçar o perfil de um paciente amputado transfemural. Para isso, foram aplicados os testes Short Form-36 (SF-36), Timed Up and Go (TUG), Questionário Nórdico de Sintomas Osteomusculares e Body Image Scale (BIS) em uma paciente, com este nível de amputação, do Hospital Universitário de Santa Maria, localizado na cidade de Santa Maria, RS, Brasil. Aprovado sob o número de parecer 3.559.580. A paciente foi esclarecida sobre todos os protocolos e assinou o termo de livre consentimento esclarecido.

Para avaliar a qualidade de vida foi utilizado o questionário SF-36, que é uma ferramenta de avaliação é multidimensional, formada por 36 itens distribuídos em oito domínios: capacidade funcional, aspectos físicos, dor, estado geral da saúde, vitalidade, aspectos sociais, aspectos emocionais e saúde mental. Apresenta um escore final que varia de zero a 100, no qual zero corresponde ao pior nível de qualidade de vida e 100 ao melhor. ${ }^{5}$

O questionário Body Image Scale (BIS), foi aplicado para percepção e satisfação com a imagem corporal. Esta ferramenta foi originalmente desenvolvida para avaliar a satisfação com a imagem corporal de pacientes com qualquer tipo de câncer, que foi adaptado para indivíduos amputados por meio da substituição dos termos "doença" e "tratamento" por "amputação". ${ }^{6}$ Este instrumento apresenta dez itens sobre aspectos de afetividade, comportamento e crenças a respeito do próprio corpo e das modificações causadas pela amputação. As opções de resposta variam de zero (nada) a três (muito) e o escore final pode variar de zero a 30 , desse modo, quanto mais baixa esta pontuação, é indicativo de menores sofrimentos em relação ao próprio corpo. ${ }^{7}$

O Timed Up and Go (TUG) é um teste de avaliação de mobilidade, cujo desempenho está relacionado com o equilíbrio, marcha e capacidade funcional, constituindo um instrumento que também avalia o risco de quedas. ${ }^{8,9}$

O Questionário Nórdico de Sintomas Osteomusculares é composto por uma figura humana em posição ortostática posterior divido em nove regiões. A partir desta imagem, o indivíduo responde "sim" mediante a presença como dor, formigamento ou dormência. O questionário ainda contém questões que se referem a desconfortos osteomusculares nas regiões representadas, levando em considerando os últimos doze meses e sete dias e se, como consequências destas dores, houve impedimento de realizar as atividades de vida diária normais ou se o 
indivíduo procurou algum profissional da saúde nos últimos doze meses devido a esta condição. ${ }^{10}$

\section{RELATO DE CASO}

Paciente I.F.M.A., sexo feminino, amputada transfemural esquerda, 48 anos, $98 \mathrm{~kg}, 1,65 \mathrm{~m}$, Índice de Massa Corporal (IMC) 35,99 (obesidade grau II), fumante, aposentada e sedentária. Sua amputação é de etiologia traumática decorrente de um acidente de motocicleta no qual foi atingida por uma caminhonete há 15 anos. Na ocasião, além da amputação, teve fratura na cintura pélvica e a uretra seccionada, fator que desencadeou incontinência urinária.

I.F.M.A. chegou ao serviço de fisioterapia do Hospital Universitário de Santa Maria (HUSM) no mês de abril de 2018 fazendo uso de muletas axilares para seu deslocamento. Durante a primeira avaliação, relatou possuir duas próteses confeccionadas, no entanto, não consegue utilizá-las, já que não se adaptou a nenhuma devido à alteração anatômica da pelve decorrente do acidente. Informou não utilizar nenhum medicamento ou possuir patologia associada, além da incontinência urinária.

A paciente não realiza o enfaixamento do coto em sua rotina, o que o torna flácido. Sua cicatriz está em bom estado, sem aderências. Seu coto possui a pele íntegra, porém, apresenta sensibilidade prejudicada na região posterior. Queixa-se de desconforto na articulação do quadril do membro direito, dor na coluna lombar e dor no membro superior direito na região do epicôndilo lateral. Além da anamnese e exame físico, foram aplicados questionários e testes buscando conhecer o perfil da paciente.

\section{RESULTADOS E DISCUSSÃO}

Para avaliar a paciente foi utilizado $\mathrm{o}$ questionário de qualidade de vida SF-36. A média geral da paciente neste questionário foi de 70,75, e a pontuação final de cada domínio está representada na figura 1.

Conforme oafigura 1, percebe-se quea paciente obteve pontuação melhor nos domínios de limitação por aspectos físicos (100) e aspectos emocionais (100), o que indica uma adaptação da paciente em relação à amputação. Na avaliação da qualidade de vida dentro do domínio capacidade funcional conseguiu uma pontuação de 45 , representando o valor mais baixo dentro do teste. Na dimensão de vitalidade, obteve uma média de pontuação de 60 . Já no domínio dor e saúde mental alcançou uma pontuação média igual nos dois (72). No aspecto estado geral de saúde obteve 67 pontos como média e em aspectos sociais sua pontuação foi de 50. Essas alterações na qualidade de vida em pessoas amputadas são justificadas pelas dificuldades que os mesmos possuem com com as habilidades básicas e tarefas diárias, dessa forma

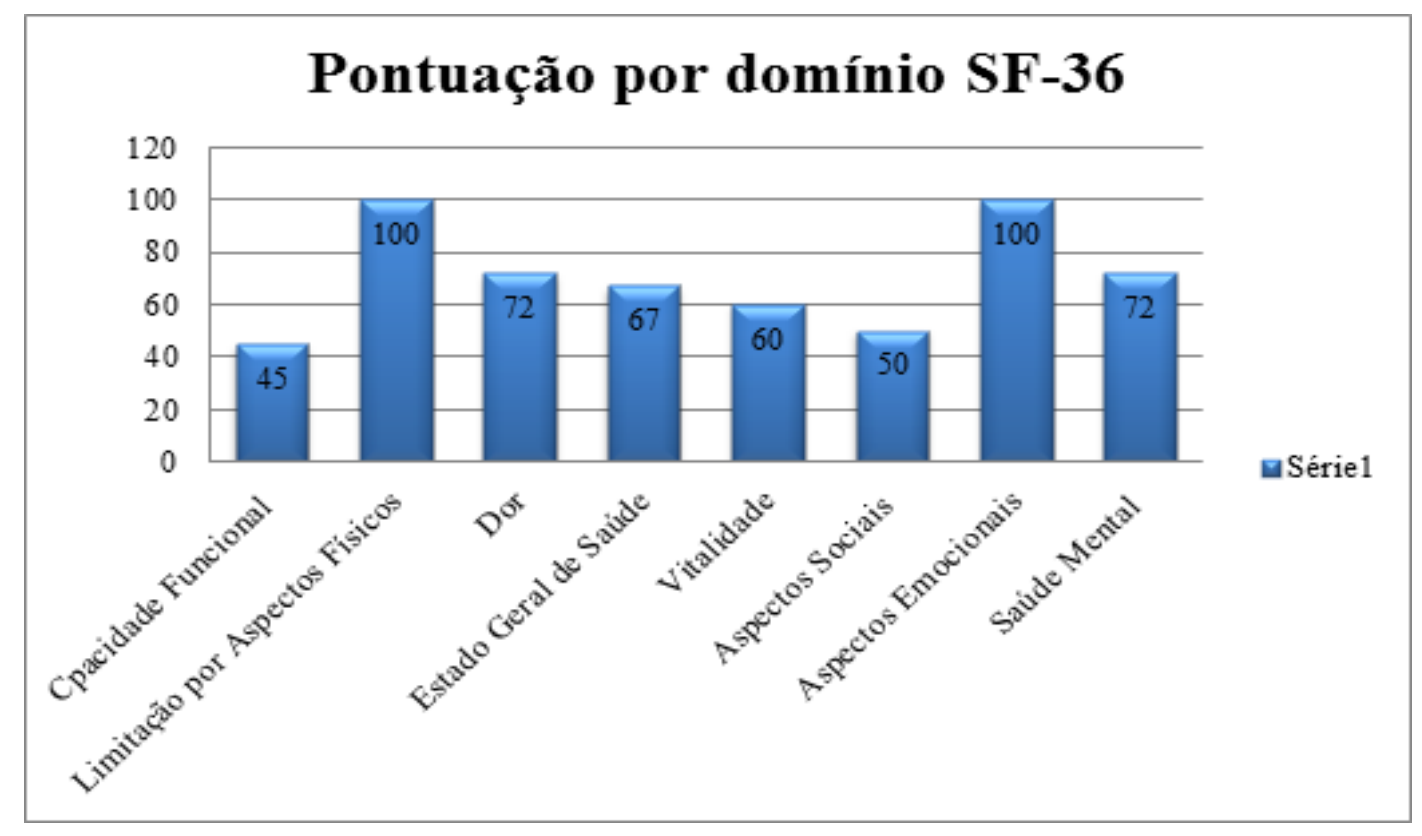

Figura 1 - pontuação por domínios do SF 36. 
gerando mudanças na vida profissional, pessoal e afetiva. Isso pode acarretar no desenvolvimento de sentimentos de inferioridade, revolta, raiva, choque e falta de aceitação consigo mesmo. ${ }^{11}$

No quesito percepção e satisfação com a imagem corporal, foi aplicado o questionário Body Image Scale (BIS). Neste teste, I.F.M.A. teve como resultado 17 pontos, correspondente a uma média satisfação com a imagem corporal e sofrimento em relação ao próprio corpo. Neste aspecto, a paciente referiu inúmeras vezes insatisfação corporal, principalmente em relação ao seu peso, e relatou que isto interfere em suas relações afetivas. Isto é justificável visto que com a perda de uma parte do corpo o indivíduo tem uma brusca alteração da imagem corporal, sendo este um processo difícil e doloroso, que impõe um novo modo de viver e de se relacionar. Dessa forma, tendo o paciente que se adaptar, readaptar, aprender a viver novamente, assumindo uma nova perspectiva do mundo para si e para os outros. $^{12}$

NoTimedUpand Go(TUG) a pacientedemorou 28 segundos para percorrer o trajeto proposto, com o auxílio das muletas axilares, representando um risco de quedas moderado. Sabe-se que a amputação resulta em perdas articulares, inserções e massa muscular e informações proprioceptivas ${ }^{13}$ que irão impactar no equilíbrio e, consequentemente, na mobilidade do indivíduo. Para que a manutenção do equilíbrio seja eficiente, faz-se necessário que muitos elementos estejam íntegros, como o sistema musculoesquelético, nervoso, proprioceptivo, visual e vestibular. Com a amputação, alguns destes mecanismos são lesados e dessa forma, o equilíbrio em amputados de membros inferiores é comprometido. Por estas razões, avaliar o equilíbrio do sujeito amputado é fundamental para a reabilitação, porque a redução do mesmo vai acarretar medo de cair, restrição de mobilidade, quedas e suas consequências físicas e psicológicas. ${ }^{14}$

No Questionário Nórdico de Sintomas Osteomusculares a amputada relatou sentir dor nos últimos doze meses em cotovelos, lombar e quadril. Devido à dor na região do cotovelo e quadril relata ter sido impedida de realizar atividades normais como atividades domésticas e trabalho. Também informou que não procurou nenhum outro profissional da área da saúde para solucionar estas algias. Nos últimos sete dias anteriores à avaliação, relatou sentir dor na articulação do quadril do membro direito.

As queixas de dor e desconforto apresentadas pela paciente estão de acordo com o que é descrito para seu quadro clínico, pois é visto que a sobrecarga no membro contralateral do amputado unilateral de membro inferior é severa e constante, principalmente quando o indivíduo não é protetizado e, portanto, faz uso de dispositivos auxiliares, como muletas, para a marcha. Ademais, a falta de harmonia na distribuição do peso e alinhamento corporal após a amputação são fatores que aumentam o ônus para o membro íntegro, que pode vir a desenvolver disfunções como osteoartrite. ${ }^{4,15,16}$ Além disso, é comum sujeitos com amputações de membros inferiores reportarem desconforto em outras regiões, como a lombar. ${ }^{4,15}$

Dessa forma, ressalta-se que os resultados encontrados evidenciam a importância de um olhar mais abrangente acerca do paciente amputado, indo além da abordagem pragmática, sendo indispensável humanizar a relação terapeuta paciente.

\section{CONCLUSÃO}

Conclui-se que I.F.M.A. com amputação transfemural apresenta risco de quedas moderado e suas queixas de dor e desconforto estão de acordo com o que é descrito para seu quadro clínico. A qualidade de vida está em bons níveis devido a uma provável adaptação da paciente a sua condição, e, sobre sua imagem corporal, a pontuação obtida refletiu um sofrimento moderado em relação ao próprio corpo. A partir dos resultados, percebe-se que a amputação não afeta somente aspectos físicos, mas tem reflexos no indivíduo em sua totalidade. Neste caso descrito, os aspectos emocionais decorrentes da amputação eram mais preponderantes de forma negativa do que os aspectos físicos na vida da paciente.

Por mais que a fisioterapia tenha um enfoque principalmente na reabilitação física, é preciso perceber que o paciente não alcançará uma evolução satisfatória se apresentar comprometimentos em outras áreas, como a psicossocial. Sendo assim, é imprescindível que a avaliação fisioterapêutica seja integral, ou seja, que contemple todos os aspectos da vida do sujeito, assim trazendo como consequência uma terapêutica efetiva e mais individualizada.

Como limitação do estudo, percebeuse a escassez de literatura no que diz respeito à amputação transfemural. Dessa forma, para a realização deste trabalho, houve grande dificuldade em encontrar na literatura científica referências atuais e testes específicos para a população amputada que 
quantifiquem variáveis como imagem corporal e dor. Portanto, faz-se necessários novos estudos com esta população não somente para suprir a carência citada acima, mas porque é de fundamental importância que os profissionais da área da saúde que irão trabalhar com estes pacientes identifiquem as possíveis consequências de uma amputação.

Ainda, a partir dos resultados encontrados neste estudo, podemos evidenciar e concluir a importância de um olhar mais abrangente acerca do paciente amputado, o qual deve ir além dos procedimentos e da abordagem pragmática, abordando também de forma indispensável uma relação humanizada entre o profissional da área da saúde e o paciente.

\section{REFERÊNCIAS}

1. Carvalho J. Amputações de membros inferiores: em busca da plena reabilitação. São Paulo: Manole; 2003.

2. Ministério da Saúde. Secretaria de Atenção à Saúde. Departamento de Ações Programáticas Estratégicas. Diretrizes de atenção à pessoa amputada. Brasília; 2012. p. 7.

3. O’Sullivan S; Schmitz T. Fisioterapia Avaliação e Tratamento.

4. ed. São Paulo: Manole; 2004.

4. Abdalla AA, Galindo J, Ribeiro SC, Riedi C, Ruaro JA, Fréz AR. Correlação entre qualidade de vida e capacidade locomotora de indivíduos com amputação de membros inferiores. ConScientiae Saúde 2013,12(1):106-13. doi: https:// doi.org/10.5585/ConsSaude.v12n1.4002

5. Ciconelli RM, Ferraz M, Santos W, Meinão I, Quaresma MR. Tradução para a língua portuguesa e validação do questionário genérico de avaliação de qualidade de vida SF-36 (BrasilSF-36). Rev. Brasileira de reumatologia 1999, 39(3):143-50.

6. Moreira H, Silva S, Marques A, Canavarro M. The portuguese version of the body image scale (bis) - psychometric properties in a sample of breast cancer patients. European Journal of Oncology Nursing 2010, 14(2): 111-18. doi: https://doi. org/10.1016/j.ejon.2009.09.007

7. Hopwood P, Fletcher I, Lee A, Al Ghazal S. A body image scale for use with cancer patients. European Journal of Cancer 2001,37(2):18997. doi: https://doi.org/10.1016/s09598049(00)00353-1

8. Chamlian TR, Melo ACO. Avaliação funcional em pacientes amputados de membros inferiores. Acta Fisiátrica 2008, 15(1):49-58.

9. Silveira JF, Lima KB, Goulartet CL, Adolfo JR, Cardoso DM, Silva ACF, Paiva DN. Avaliação da capacidade funcional, força muscular e função pulmonar de pacientes amputados e protetizados ao nível transfemural: estudo piloto. Cinergis 2015,16(1)01-04. doi: http://doi.org/10.17058/cinergis. v16i1.4800
10. Descatha A, Roquelaure Y, Chastang J, Evanoff B, Melchior M, Mariot C, Ha C, Imbernon E, Goldberg M, Leclerc A. Validity of Nordic-style questionnaires in the surveillance of upperlimb work-related musculoskeletal disorders. Scand J Work Environ Health 2007,33(1):58-65. doi; http://doi.org/10.5271/ sjweh.1065

11. Senra H, Oliveira RA, Leal I, Vieira C. Beyond the body image: a qualitatie study on how adults experience lower limb amputatin. Clin Rehabil 2012;26(2):180-91. doi: http://doi. org/10.1177/0269215511410731

12. Chini GC, Boemer MR. A amputação na percepção de quem a vivencia: um estudo sob a ótica fenomenológica. Rev. Latino-Am. Enfermagem 2007, 15(2): 330-6. doi: https://doi. org/10.1590/S0104-11692007000200021

13. Ribeiro MP. Influência do nível de amputação no equilíbrio estático de amputados traumáticos. EFDeportes.com Revista Digital 2013,18(179).

14. Baraúna MA, Duarte FII, Sanchez HM, Canto RST; Malusá S; Campelo-Silva CD, Ventura-Silva RA. Avaliação do equilíbrio estático em indivíduos amputados de membros inferiores através da biofotogrametria computadorizada. Revista Brasileira de Fisioterapia 2006,10(1). doi: https://doi.org/10.1590/S141335552006000100011

15. Machado VI, Roque V, Pimentel S, Rocha A, Duro H. Caracterização psicossocial de uma população portuguesa de amputados do membro inferior. Acta Médica Portuguesa 2012,25(2):77-82.

16. Chamlian TR, Santos JK, Faria CC, Pirrelo M, Leal C. Dor relacionada à amputação e funcionalidade em indivíduos com amputações de membros inferiores. Acta Fisiátrica 2014,21(3):113-116.
Recebido em: 28/03/2021

Aceito em:23/04/2021

Como citar: DALENOGARE, Jéssica Franco et al. Perfil clínico de um paciente amputado transfemoral: relato de caso. Revista Interdisciplinar de Promoção da Saúde, Santa Cruz do Sul, v. 3, n. 3, jul 2020. ISSN 2595-3664. Disponível em: <https://online. unisc.br/seer/index.php/ripsunisc/article/view/16360>. Acesso em: 01 jul 2020. doi:https://doi.org/10.17058/rips.v3i3.16360 